[cited 2021 Jan 13]. https:/ / www.wsj.com/articles/ u-k-delays-second-covid-19-vaccine-dose-as-europeponders-how-to-speed-up-immunization-11609334172

8. US Food and Drug Administration. FDA statement on following the authorized dosing schedules for COVID-19 vaccines. January 4, 2021 [cited 2021 Jan 14]. https:/ / www. fda.gov/news-events/press-announcements/fda-statementfollowing-authorized-dosing-schedules-covid-19-vaccines

9. Livingston EH. Necessity of 2 doses of the Pfizer and Moderna COVID-19 vaccines. JAMA. 2021;325:898. 10.1001/ jama.2021.1375 https://doi.org/10.1001/jama.2021.1375

10. Science advisers: publish evidence behind COVID vaccine dosing strategy. Nature. 2021;589:169-70. https://doi.org/10.1038/d41586-021-00045-8

Address for correspondence: Lauren Ancel Meyers, Department of Integrative Biology, 1 University Station C0990; Austin, TX 78712, USA; email: laurenmeyers@austin.utexas.edu

\section{SARS-CoV-2 Aerosol Exhaled by Experimentally Infected Cynomolgus Monkeys}

\author{
Chunmao Zhang, ${ }^{1}$ Zhendong Guo, ${ }^{1}$ \\ Zongzheng Zhao, ${ }^{1}$ Tiecheng Wang, Liang Li, \\ Faming Miao, Cheng Zhang, Yuanguo Li, Yuwei Gao \\ Author affiliations: College of Veterinary Medicine at Hebei \\ Agricultural University, Baoding, China. (C. Zhang); Military \\ Veterinary Research Institute, Changchun, China (C. Zhang, \\ Z. Guo, Z. Zhao, T. Wang, L. Li, F. Miao, C. Zhang, Y. Li, Y. Gao)
}

DOI: https://doi.org/10.3201/eid2707.203948

We analyzed size of severe acute respiratory coronavirus 2 (SARS-CoV-2) aerosol particles shed by experimentally infected cynomolgus monkeys. Most exhaled particles were small, and virus was mainly released early during infection. By postinfection day 6 , no virus was detected in breath, but air in the isolator contained large quantities of aerosolized virus.

\begin{abstract}
A lthough airborne transmission of severe acute respiratory syndrome coronavirus 2 (SARS-CoV-2) has been proven possible among humans (1), cats (2), ferrets (3), and Syrian hamsters (4), the relative roles of droplets and aerosols in the airborne transmission
\end{abstract}

${ }^{1}$ These authors contributed equally to this article. of SARS-CoV-2 remain controversial. A recent study showed that coronavirus disease (COVID-19) patients exhaled millions of SARS-CoV-2 particles during early infection stages (5). However, the size distribution of SARS-CoV-2 aerosol particles in exhaled breath of COVID-19 patients is not clear.

To analyze size distribution of SARS-CoV-2 aerosols shed by cynomolgus monkeys, we inoculated 3 monkeys with SARS-CoV-2 via a combination of intranasal, intratracheal, and ocular routes. Monkeys were kept in individual cages placed in an isolator (biosafety housing with HEPA filters and independent ventilation system). The exhaled breath and air in the isolator were collected by a 6-stage Andersen sampler (https://tisch-env.com) at postinfection days 2,4 , and 6 , and we quantified the viral RNA copies in samples (Appendix, https://wwwnc.cdc.gov/ EID/article/27/7/20-3948-App1.pdf). We also determined size distribution of SARS-CoV-2 particles.

The virus particles monkeys exhaled peaked at postinfection day 2 and ranged from 11,578 to 28,336 RNA copies during a 40-minute period. On average, each monkey exhaled 503 virus particles/min and 209.5 virus particles/L of exhaled breath. At postinfection day 4 , the number of exhaled virus particles decreased substantially, ranging from 3,369 to 5,134 RNA copies during a 40-minute period. On average, each monkey exhaled 106 virus particles/min and 44 virus particles/L of breath. At postinfection day 6, no viral RNA was detected in exhaled breath (Figure, panel A; Appendix Figure 1). At postinfection days 2, 4, and 6, viral RNA was detected in air within the isolator housing the monkeys; we detected 6,182-13,608 RNA copies during a 30-minute period (Figure, panel C).

We measured size distribution of SARS-CoV-2 aerosol particles shed by the monkeys. In exhaled breath of inoculated monkeys and in air in the isolator, viral RNA was detected in all size bins, $0.65-2.1 \mathrm{~mm}$, 2.1-4.7 $\mathrm{mm}$, and $>4.7 \mathrm{~mm}$, at postinfection days 2 and 4; most were concentrated in the 2.1-4.7-mm bin (Figure, panels B, D; Appendix Tables 1, 2). For exhaled breath, virus particles in each of the 3 size bins accounted for $27.4 \%, 49.6 \%$, and $23.0 \%$ of the total virus copies $/ 40$ $\mathrm{min}$, respectively; for air in the isolator, virus particles in each of the 3 size bins accounted for $3.8 \%, 75.0 \%$, and $21.2 \%$ of the total virus copies/30 $\mathrm{min}$, respectively (Appendix Tables 1, 2, Figure 3). Most virus particles were in the smaller particle size range $(0.65-4.7 \mathrm{~mm})$, accounting for $77 \%$ to $79 \%$ of the total virus particles shed by the monkeys; droplets $(>4.7 \mathrm{~mm})$ accounted for $\approx 21 \%-23 \%$ (Appendix Tables 1, 2, Figure 3). We tried to isolate live virus by sequentially passaging 

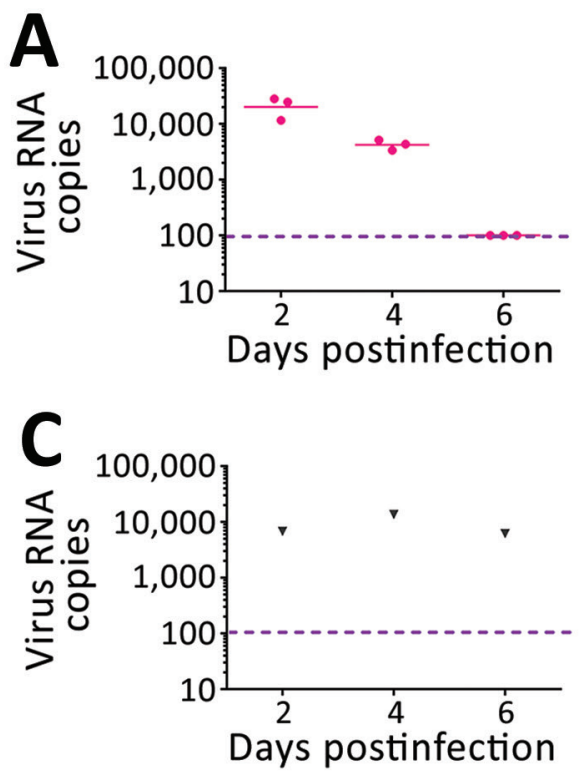
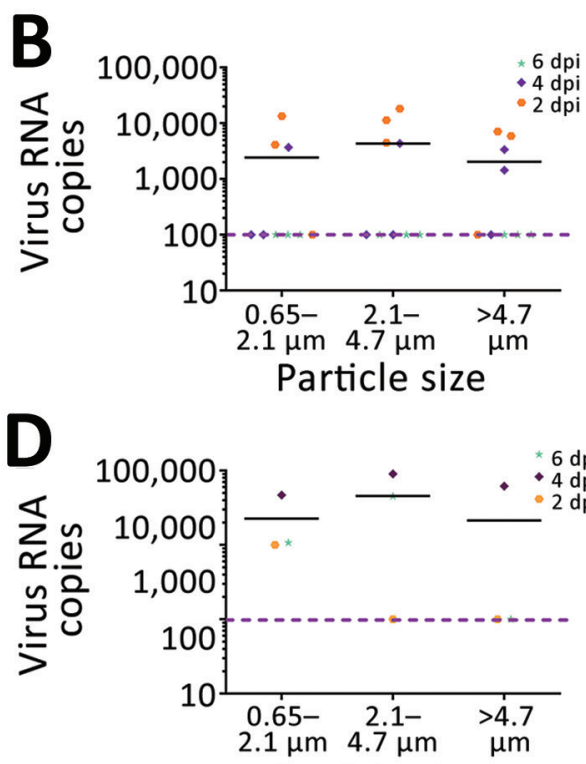

Figure. Viral RNA copies and size distribution of severe acute respiratory syndrome coronavirus 2 aerosols shed by experimentally infected cynomolgus monkeys. A) Viral RNA copies in aerosols directly expelled during 40 minutes of breathing. B) Size distribution of virus aerosols directly expelled during 40 minutes of breathing. C) Viral RNA copies in aerosols from the housing isolator during 30 minutes of sampling. D) Size distribution of virus aerosols in the isolator during 30 minutes of sampling. dpi, days postinfection. The pink dotted line indicates the limit of detection. these samples in Vero-E6 cells 3 times (Appendix) but obtained no live virus and observed no cytopathic effects; the reasons for this failure are unknown.

The World Health Organization cites the 2 main transmission routes of SARS-CoV-2 as large respiratory droplets and contact transmission. However, we found that monkeys infected with SARS-CoV-2 emitted large quantities of virus aerosol particles, most of which were smaller $(<4.7 \mu \mathrm{m})$. Ma et al. showed that COVID-19 patients exhaled millions of SARSCoV-2 particles/hour (5), far more than that noted for monkeys. This variation may result from biological differences between humans and monkeys and different sampling methods. Respiration is much slower in monkeys $(2.4 \mathrm{~L} / \mathrm{min})$ than in humans $(12 \mathrm{~L} / \mathrm{min})$. In addition, during sampling, monkeys were anesthetized and breathed slowly through their nostrils, possibly emitting fewer virus particles than when awake. The size of airborne particles determines how the virus is transmitted. Droplets $(>4.7 \mu \mathrm{m})$ can travel limited distances; smaller particles $(<4.7 \mu \mathrm{m})$ stay airborne longer and spread widely $(6,7)$. Our findings suggest that aerosol transmission might contribute to SARS-CoV-2 spread. Personal protection requires wearing face masks, maintaining social distancing, and reducing gatherings. Infection risk in enclosed spaces is lowered by natural wind or mechanical airflow ventilation.

Cynomolgus monkeys infected with SARSCoV-2 emitted most virus particles in early infection stages; particles decreased substantially at postinfection day 6. Zhou et al. demonstrated that COVID-19 patients emitted fewer virus particles when they were recovering and ready for discharge than did those in early infection stages (8). At postinfection day 6 , no virus was detected in the breath of monkeys, but air in the isolator housing the monkeys still contained large quantities of aerosolized virus. These different seemingly noncoherent observations can be attributed to monkey activity, air flow, and some virus aerosol residues exhaled by monkeys for a relatively long period before sampling. Recently, Asadi et al. showed that aerosolized fomites (microscopic particles) played a role in influenza virus transmission between guinea pigs (9). SARS-CoV-2 may be carried and transmitted between humans by aerosolized fomites. Most SARS-CoV-2 aerosol particles exhaled by the cynomolgus monkeys in this study were smaller, suggesting that aerosols might be a route for SARS-CoV-2 transmission.

\section{Acknowledgments}

We thank the staff at the Biosafety Level 3 laboratories of Military Veterinary Research Institute for their support and help.

This research was supported by the National Natural Science Foundation of China (32000134) and the National Major Research and Development Program (2020YFC0840800).

\section{About the Author}

Drs. Chunmao Zhang, Guo, and Zhao are investigators at the Military Veterinary Research Institute. Their primary interests are pathogenicity and airborne transmissibility of respiratory viruses, especially influenza viruses. 


\section{References}

1. Anderson EL, Turnham P, Griffin JR, Clarke CC. Consideration of the aerosol transmission for COVID-19 and public health. Risk Anal. 2020;40:902-7. https:/ / doi.org/ 10.1111/risa. 13500

2. Shi J, Wen Z, Zhong G, Yang H, Wang C, Huang B, et al. Susceptibility of ferrets, cats, dogs, and other domesticated animals to SARS-coronavirus 2. Science. 2020;368:1016-20. https://doi.org/10.1126/science.abb7015

3. Kim YI, Kim SG, Kim SM, Kim EH, Park SJ, Yu KM, et al. Infection and rapid transmission of SARS-CoV-2 in ferrets. Cell Host Microbe. 2020;27:704-709.e2. https:/ / doi.org/ 10.1016/j.chom.2020.03.023

4. Sia SF, Yan LM, Chin AWH, Fung K, Choy KT, Wong AYL, et al. Pathogenesis and transmission of SARS-CoV-2 in golden hamsters. Nature. 2020;583:834-8. https:/ / doi.org/ 10.1038/s41586-020-2342-5

5. Ma J, Qi X, Chen H, Li X, Zhang Z, Wang H, et al. Coronavirus disease 2019 patients in earlier stages exhaled millions of severe acute respiratory syndrome coronavirus 2 per hour. Clin Infect Dis. 2020;ciaa1283. https://doi.org/10.1093/cid/ciaa1283

6. Bischoff WE, Swett K, Leng I, Peters TR. Exposure to influenza virus aerosols during routine patient care. J Infect Dis. 2013;207:1037-46. https://doi.org/10.1093/ infdis/jis773

7. Brankston G, Gitterman L, Hirji Z, Lemieux C, Gardam M. Transmission of influenza $A$ in human beings. Lancet Infect Dis. 2007;7:257-65. https:/ / doi.org/10.1016/ S1473-3099(07)70029-4

8. Zhou L, Yao M, Zhang X, Hu B, Li X, Chen H, et al. Breath-, air- and surface-borne SARS-CoV-2 in hospitals. J Aerosol Sci. 2021;152:105693. https:// doi.org/10.1016/ j.jaerosci.2020.105693

9. Asadi S, Gaaloul Ben Hnia N, Barre RS, Wexler AS, Ristenpart WD, Bouvier NM. Influenza A virus is transmissible via aerosolized fomites. Nat Commun. 2020;11:4062. https:/ / doi.org/10.1038/s41467-020-17888-w

Address for correspondence: Yuwei Gao, Military Veterinary Research Institute, 666 Liuying West Rd, Changchun, 130122, China; email:gaoyuwei@gmail.com

\section{Possible Human-to-Dog Transmission of SARS-CoV-2, Italy, 2020}

\author{
Nicola Decaro, Gabriele Vaccari, Alessio Lorusso, \\ Eleonora Lorusso, Luca De Sabato, \\ Edward I. Patterson, Ilaria Di Bartolo, Grant L. Hughes, \\ Liana Teodori, Costantina Desario, Barbara Colitti, \\ Dominga Ricci, Domenico Buonavoglia, Sergio Rosati, \\ Vito Martella, Cesare Cammà, Umberto Agrimi, \\ Gabriella Elia
}

\begin{abstract}
Author affiliations: University of Bari, Valenzano, Italy (N. Decaro, E. Lorusso, C. Desario, D. Buonavoglia, V. Martella, G. Elia); Istituto Superiore di Sanità, Rome, Italy (G. Vaccari, L. De Sabato, I. Di Bartolo, U. Agrimi); Istituto Zooprofilattico Sperimentale dell'Abruzzo e del Molise "G. Caporale," Teramo, Italy (A. Lorusso, L. Teodori, C. Cammà); Liverpool School of Tropical Medicine, Liverpool, UK (E.I. Patterson, G.L. Hughes); University of Turin, Turin, Italy (B. Colitti, S. Rosati); Ambulatorio Veterinario Dott.ssa Ricci Dominga, Andria, Italy (D. Ricci)
\end{abstract}

DOI: https://doi.org/10.3201/eid2707.204959

We detected severe acute respiratory syndrome coronavirus 2 in an otherwise healthy poodle living with 4 family members who had coronavirus disease. We observed antibodies in serum samples taken from the dog, indicating seroconversion. Full-length genome sequencing showed that the canine and human viruses were identical, suggesting human-to-animal transmission.

C oronavirus disease (COVID-19), caused by infection with severe acute respiratory syndrome coronavirus 2 (SARS-CoV-2), emerged in humans in $\mathrm{Wu}-$ han, China, in late December 2019, probably because of spillover from an unidentified animal host (1). Dogs and cats, to which some coronaviruses are endemic (2), are also susceptible to SARS-CoV-2 infection $(3,4)$. Although the spread of SARS-CoV-2 is maintained mainly by human-to-human transmission, the epidemiologic implications of animal susceptibility remain uncertain (4). We characterized the full genome of a SARS-CoV-2 isolate detected in a dog.

A female poodle, who was 1.5 years of age, lived with 4 family members in Bitonto, Italy. All family members had signs and symptoms of COVID-19, the illness caused by SARS-CoV-2 infection. High temperature $\left(37.5^{\circ} \mathrm{C}-38.5^{\circ} \mathrm{C}\right)$, coughing, anosmia, and ageusia developed in the mother, who was 54 years of age, on October 31, 2020. The woman tested positive for SARS-CoV-2 by a rapid antigen test conducted on November 3, 2020. The local health authority 